\title{
Multicast Wavelength Assignment with Sparse Wavelength Converters to Maximize the Network Capacity using ILP formulation in WDM Mesh Networks
}

\author{
I-Shyan Hwang, San-Nan Lee, student member, IEEE, and Ying-Fung Chuang \\ Department of Computer Engineering and Science \\ Yuan-Ze University, Chung-Li 32026, Taiwan \\ E-mail: ishwang@saturn.yzu.edu.tw, grand@saturn.yzu.edu.tw
}

\begin{abstract}
In general, the multicast routing and wavelength assignment (MC-RWA) can be subdivided as routing and wavelength assignment issues in WDM mesh networks. Previous studies on WDM multicast have mainly focused on the WDM multicast routing. The multicast wavelength assignment problem is studied in this paper. A unicast routing path can be established by a lightpath in an all-optical network. However, in multicasting case, a multicast routing tree can be established by a single light-tree or several lightpaths, or a combination of several light-trees and lightpaths. We propose a wavelength assignment algorithm for finding an optimal combination of lightpaths and light-trees to construct a newly arrived multicast session. First of all, two cost functions are given to evaluate the establishing cost for each feasible wavelength, and then find a set of wavelength that covers all destinations with the minimal cost using Integer Linear Program (ILP) formulation.
\end{abstract}

Keywords: multicast routing and wavelength assignment (MC-RWA), network capacity, integer linear program (ILP), optical networks.

\section{Introduction}

As demand for bandwidth in today's Internet is increasing every day, wavelength-routed alloptical networks are considered to be candidates for the next generation wide-area backbone networks. In an all-optical network with wavelength-division multiplexing (WDM) transmitttssion, each data channel is carried on a unique wavelength (or optical frequency) and a single optical fiber has many different wavelengths. There are two important types of WDM networks: wavelength-selective (singlehop) network and wavelength-interchangeable (multihop) network [1]. In wavelength-selective networks, each message is transmitted from the source to the destination without any opticalto-electronic conversion within the network. It can be realized by using a single wavelength to establish a connection. A critical measure for improving network performance of WDM networks is the wavelength conversion. The network employing the technique of wavelength conversion is known as wavelengthinterchangeable network, and it has been shown that a relatively small number of converters can achieve satisfactory performance in [2]. In wavelength-interchangeable networks, a message entering a wavelength convertible router (or wavelength converter) on a particular wavelength can be converted into the electronic medium by a receiver and retransmitted on a new wavelength by a transmitter. This paper examines wavelength-interchangeable networks. Eliminating wavelength conversion reduces significantly the cost of the switch; however it may lead to reduction in network efficiency. Multicasting in a network is needed for a number of applications where a session requires connectivity between more than two participants, and is increasingly popular on the Internet.

In the literature, wavelength assignment problems for multicast have been studied recently. For example, [3] proposed an algorithm for minimizing the number of wavelength conversions. [4,5] addressed the problems for maximizing the number of wavelengths. In [6], a model of wavelength assignment to maximize the network capacity (WA-MNC) is introduced, and [7] implemented the WA-MNC for multicasting in a special regular topology, bidirectional ring networks. A dynamic centralized algorithm using WA-MNC for unicast named Relative Least Influence algorithm (RLI) is proposed in [8].

In this paper, we study the WA-MNC for multicast on an arbitrary mesh network topology. A heuristic algorithm is proposed to maximize the total number of users served in a multicast session and minimize the effect on the network after this assignment. First, we find the maximum number of users that can be served in a multicast session. Second, we assign the wavelengths to the multicast session such that the effect on the network is minimized. Since WA-MNC works on a single-hop network, the presence of wavelength converters must be considered. Thus, we continue using the wavelength converter placement algorithm proposed in [9] for the sparse wavelength conversion case; it places wavelength converters 
such that the whole network is divided into several all-optical networks.

The rest of the paper is organized as follows. In Section 2, we first define the model under consideration, and then we present our wavelength assignment algorithm using WA-MNC for multicasting. Finally, Section 3 concludes the paper and presents some possible future work.

\section{The ILP-based Wavelength Assignment Algorithm}

In this section, the ILP-based wavelength assignment (ILPWA) algorithm is described in detail. The optical networks can be represented by an undirected graph $G=(V, E)$ where $V=\left\{v_{1}, v_{2}, \ldots, v_{N}\right\}$ is the set of vertices and $E$ is the set of edges. Let $N$ be the number of vertices in $G$ such that $|V|=N$. We define a wavelength as "available" on a path if that wavelength is not used on any of the links constituting the path. Otherwise, a wavelength is "unavailable" on a path. A "partition" is a subgraph of $G$ and this subgraph represents a single-hop network.

Since WA-MNC for multicast is NP-hard. There is no optimal solution to the problem in reasonable time. We develop a heuristic multicast wavelength assignment algorithm in an arbitrary mesh network, and use integer linear programming (ILP) to find an optimal solution.

Let $r=<s, D, T>$ be the newly arrived multicast request, where $s$ is the multicast source, $D$ is the destination set, and $T=\left(V^{\prime}, E^{\prime}\right)$ is an auxiliary graph that represents a multicast routing tree.

$\Lambda(T)=\left\{\lambda_{k} \mid \lambda_{k}\right.$ can be assigned to $T$ that covers at least one destination $\}$. Let $D^{\prime}$ be the set of users that can be served in the newly arrived request $r . c_{k}$ is the decrease in network capacity and can be viewed as the cost of assigning wavelength $\lambda_{k}$ if $\lambda_{k}$ is assigned to $r$, and can be calculated by counting the potential paths in the whole network if $\lambda_{k}$ is assigned.

Before assigning wavelengths to a newly arrived multicast request, we have to calculate the assigning cost for each $w \in \Lambda(T)$. In [8], the cost function for assigning wavelength $\lambda_{k}$ is defined as the number of potential paths that cannot use $\lambda_{k}$ in the future. It also showed that even for multicasting, a potential multicast request with $M$ destinations should be viewed as $M$ unicasts. Therefore, we just need to assume that only unicast requests are generated in the future. While counting potential paths for a wavelength $\lambda_{k}$, we first have to derive a connected graph $G_{k}=\left(V_{k}, E_{k}\right)$ from the original network $G=(V, E)$. Starting at the source node $s$, we find a connected component in $G=(V, E)$ by determining if $\lambda_{k}$ is available in a link $e \in E$. Hence, in $G_{k}, \lambda_{k}$ is available in each link. In other words, if $\lambda_{k}$ is assigned to $T$, any potential path in $G_{k}$ that covers at least one link of $T$ cannot use $\lambda_{k}$ in the future. There are $|E|$ links in network $G$; therefore, the time complexity to derive graph $G_{k}$ is $O(|E|)$. Second, the algorithm finds all pairs of shortest paths for $G_{k}, G_{k}$ has maximum $N$ vertices, and it uses a standard single-source algorithm called Dijkstra that has a time complexity of $O\left(N^{2}\right)$ and processes $N$ times. Therefore, the algorithm for finding all pairs of shortest paths has the time complexity of $O\left(N^{3}\right)$. Finally, it calculates how many shortest paths are influenced after this assignment. Suppose there are $N$ nodes in the network, which means there are $\frac{N(N-1)}{2}$ shortest paths in the network. Therefore, the time complexity of counting how many paths are influenced after this assignment is $O\left(N^{2}\right)$, and the algorithm calculates $c_{k}$ for each wavelength $\lambda_{k}$ where $\lambda_{k} \in \Lambda$. Hence, the time complexity of the counting algorithm is $O\left(W|E| N^{5}\right)$, where $W$ is the maximum number of wavelengths in each link.

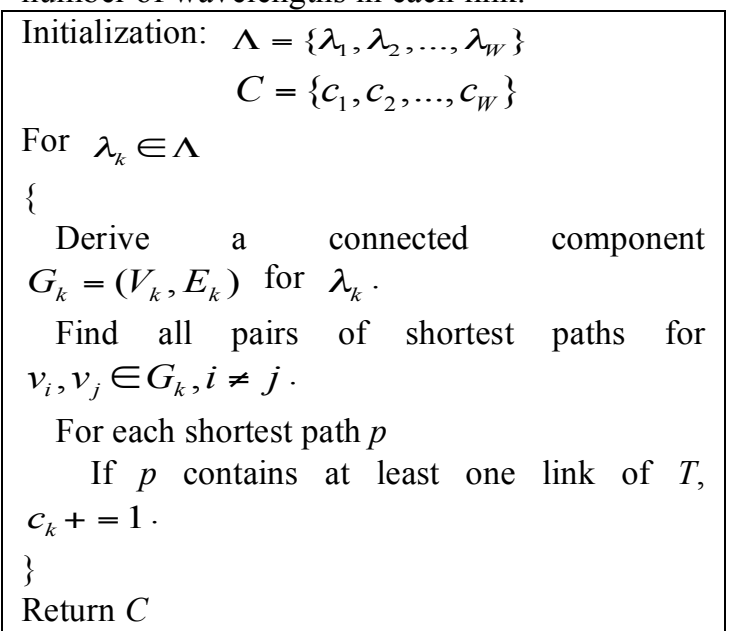

Fig. 1. Compute $c_{k}$ by counting potential connections for $G_{k}$.

In a mesh network, the complexity of counting potential paths may be too high since there are potential requests between any two 
nodes. For this reason, we develop a heuristic algorithm that only considers the number of shortest paths which are influenced after $\lambda_{k}$ is assigned to a new request. For each $G_{k}$ of $\lambda_{k}$, where $\lambda_{k} \in \Lambda$, the cost function first finds all pairs of shortest paths in $G_{k}$, and then examine how many paths in all the pairs of shortest paths are influenced after $\lambda_{k}$ is assigned to the multicast request routing tree $T$. For an undirected graph $G_{k}=\left(V_{k}, E_{k}\right)$ where $\left|V_{k}\right|=N_{k}$, there is only one shortest path between any two nodes $u$ and $v$ where $u \neq v$ and $u, v \in V$. Therefore, the number of shortest paths in a graph $G_{k}$ is $\left(\begin{array}{c}N_{k} \\ 2\end{array}\right)=\frac{N_{k}\left(N_{k}-1\right)}{2} \cdot$ On the other hand, we assume that there are only $\frac{N_{k}\left(N_{k}-1\right)}{2}$ potential requests in $G_{k}$ and counting how many requests of the $\frac{N_{k}\left(N_{k}-1\right)}{2}$ potential requests will be blocked if $\lambda_{k}$ is assigned to the newly arrived multicast request. The algorithm of counting potential connections is given in Figure 1.

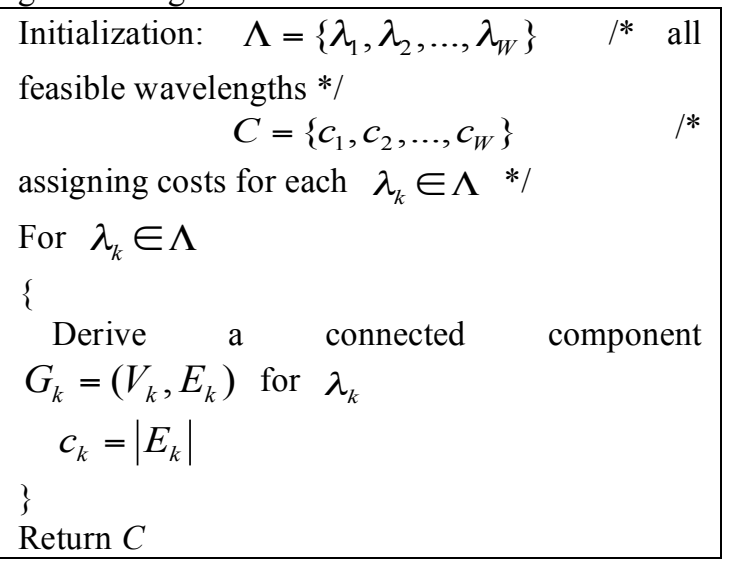

Fig. 2. Compute $c_{k}$ by counting the number of edges of $G_{k}$.

Owing to the high complexity of counting potential requests in mesh networks, we develop a simple cost function as shown in Figure. 2, where $c_{k}$ is computed by counting the edges in $G_{k}$ instead of counting potential requests which are influenced. This idea is derived from the Best-Fit memory management technique of the science of operating system, in the part of the operating system that handles memory allocation, which is called the memory manager. For example, we suppose a process requests $12 \mathrm{~KB}$ of memory and the memory manager currently has a list of unallocated blocks of $6 \mathrm{~KB}, 14 \mathrm{~KB}$,
$19 \mathrm{~KB}, 11 \mathrm{~KB}$, and $13 \mathrm{~KB}$ blocks. The Best-Fit strategy will allocate $12 \mathrm{~KB}$ of the $13 \mathrm{~KB}$ block to the process. For the MC-RWA problem formulated in this paper, a multicast request routing tree is viewed as a "process", and all $G_{k}$ of $\lambda_{k}$ where $\lambda_{k} \in \Lambda$ is viewed as "unallocated blocks". The number of edges in $G_{k}$ is viewed as the size of a block.

In an auxiliary graph $G_{k}$ of $\lambda_{k}$, there are $\frac{N_{k}\left(N_{k}-1\right)}{2}$ shortest paths in $G_{k}$. In other words, the cost function shown in Figure 1 can obtain a cost value between 1 and $\frac{N_{k}\left(N_{k}-1\right)}{2}$ to represent the establishing cost. However, the cost function given in Figure 4 just returns the number of edges $\left|E_{k}\right|$ to represent the establishing cost. Therefore, if the size of $G_{k}$ is large enough, the first cost function will yield a more accurate cost value than the second cost function. Time complexity of cost function shown in Figure 4 is $O(W|E|)$

After calculating the assigning cost for each wavelength $\lambda_{k} \in \Lambda$, we need to find a set of wavelengths and these wavelengths cover all destinations in $D^{\prime}$, such that

$$
D^{\prime}=\bigcup_{\lambda_{k} \in \Lambda} d\left(\lambda_{k}\right)
$$
where $d\left(\lambda_{k}\right) \subset D^{\prime}$ and all elements in $d\left(\lambda_{k}\right)$ are destination nodes covered by $\lambda_{k}$, and the decrease in total network capacity is minimized. In this paper, we use integer linear programming (ILP) to obtain an optimal solution. This problem is formulated as follows.

Minimize:

$$
c(r)=\sum_{\lambda_{k} \in \Lambda} c_{k} A\left(\lambda_{k}\right)
$$

\section{Subject to:}

$$
\begin{gathered}
A\left(\lambda_{k}\right)= \begin{cases}1, & \text { if } \lambda_{\mathrm{k}} \text { is assigned to } r . \\
0, & \text { otherwise. }\end{cases} \\
\sum_{\lambda_{k} \in w\left(v_{i}\right)} A\left(\lambda_{k}\right) \geq 1 \quad \text { for all } v_{i} \in D^{\prime}
\end{gathered}
$$

Wavelength_assignment $(G, r)$

\{

Calculate $c_{k}$ for each $\lambda_{k} \in \Lambda$ using algorithms given in Fig. 1 or Fig. 2.

Find the set of wavelengths that cover all destinations $D^{\prime}$ using Equations (1), (2) and (3).

For each $v_{i} \in S$

\{

Choose a wavelength $\lambda_{k}$ from $w\left(v_{i}\right)$ to cover $v_{i}$, where $\lambda_{k}$ has the minimum cost 
ratio in $w\left(v_{i}\right)$.

\}

$D-D^{\prime}$ is the users that cannot be served in the multicast session. \}

Fig. 3. Wavelength assignment algorithm using ILP formulation

For the above formulation, $c(r)$ represents the decrease in total network capacity. Equation (2) indicates that whether $\lambda_{k}$ is assigned to $r$ or not. In Equation (3), $w\left(v_{i}\right)$ represents the set of wavelengths that cover destination $v_{i}$. At least one wavelength $\lambda_{k}$ is assigned that $\lambda_{k} \in w\left(v_{i}\right)$ should be selected to cover $v_{i}$. On the other hand, a destination $v_{i}$ may be covered by two or more wavelengths according to the above wavelength selection criterion. We choose a wavelength with minimum cost ratio to cover $v_{i}$. Assume $n_{k}$ is the maximum number of destinations that wavelength $\lambda_{k}$ can reach in the multicast tree $T . \phi_{k}$ is the cost ratio of $\lambda_{k}$ and it can be calculated by $\phi_{k}=c_{k} / n_{k}$. The wavelength assignment algorithm for maximizing network capacity is given in Figure 3, $S=\left\{v_{i} \mid \sum_{\lambda_{k} \in w\left(v_{i}\right)} A\left(\lambda_{k}\right)>1\right\}$, where $S$ is the set of destinations and is covered by two or more wavelengths.

\section{Conclusion}

We have studied the multicast routing and wavelength assignment (MC-RWA) problem in this paper, and focused on maximizing the network capacity and minimizing the resources used by a multicast session. We show that a multicast wavelength assignment problem is quite different from a unicast wavelength assignment problem. For a general multicast tree, the multicast wavelength assignment to maximize the network capacity is NP-hard. We have presented a heuristic wavelength assignment algorithm with two cost functions to maximize the network capacity. The first cost function has a time complexity of $O\left(W|E| N^{5}\right)$, but it cannot get a satisfactory improvement on the second cost function, which is simply developed from the idea of the Best-Fit technique with time complexity of $O(W|E|)$

in memory management or even a worse performance with increasing traffic load. The proposed algorithm tries to find an optimal combination of lightpaths and light-trees to serve a multicast request, and aims at maximizing the network capacity and reducing the wavelength resources. We will simulate this model to prove its performance in the future.

\section{References}

[1] R. Datta, B. Mitra, S. Ghose and I. Sengupta, "An Algorithm for Optimal Assignment of a Wavelength in a Tree Topology and Its Application in WDM Networks," IEEE Journal on Selected Areas in Communications, Vol. 22, pp. 1589-1600, Nov. 2004.

[2] X. Chu, B. Li, K. Sohraby and Z. Zhang, "Routing and wavelength assignment issues in the presence of wavelength conversion for all-optical networks," 2002 Global Telecommunications Conference, Vol. 3, pp. 2787-2791, Nov. 2002.

[3] B. Chen and J. Wang, "Efficient routing and wavelength assignment for multicast in WDM networks," IEEE Journal on Selected Areas in Communications, Vol. 20, pp. 97-109, Jan 2002.

[4] X. Jia, D. Du, X. Hu, M. Lee and J. Gu, "Optimization of wavelength assignment for QoS multicast in WDM networks," IEEE Transaction on Communications, Vol. 49, pp. 341-350, Feb. 2001.

[5] X. Jia, X. Hu, L. Ruan and J. Sun, "Multicast routing, load balancing, and wavelength assignment on tree of rings," IEEE Letter on Communications, Vol. 6, pp. 79-81, Feb. 2002.

[6] S. Subramania and R. Barry, "Wavelength assignment in fixed routing WDM networks," IEEE International Conference on Communications, Vol. 1, pp. 406-410, 1997.

[7] J. Wang, B. Chen and R. N. Uma, "Dynamic wavelength assignment for multicast in all-optical WDM networks to maximize the network capacity," IEEE Journal on Selected Areas in Communications, Vol. 21, pp. 1274-1284, Oct. 2003.

[8] S. Xu, L. Li, S. Wang and C. Chen, "Wavelength assignment for dynamic traffic in WDM networks," IEEE International Conference on Net/works, 2000 (ICON 2000), pp. 375-379, Sept. 2000.

[9] V. Tamarapalli and S. H. Srinivasan, "Normalized cuts for wavelength converter placement," 2004 Workshop on High Performance Switching and Routing (HPSR), pp. 292-296, 2004. 